\title{
Epigenetic mechanism of wheat adaptation on a response to the abiotic stress
}

\author{
Minasbekyan L.A.*, Vardevanyan P.O. \\ Yerevan State University, SRI "Biology”, Department of Biophysics, Yerevan, Armenia \\ *email:minlia@ysu.am
}

Electromagnetic radiation of the radiofrequency waveband is the new anthropogenic factor of the environment and research into its influence on living organisms is of great interest. Today in Armenia there are 3 mobile network operators: Beeline, Viva Cell MTS, and U-com. The problem of electromagnetic safety becomes extremely relevant since the most technical, telecommunication, and medical devices radiate mm-wave in the range of 3-300 GHz. Thus, the territory of relatively small Armenia is covered three times by large telecommunication operators, which undoubtedly harms human health. Rearrangements in the nuclear envelope and the content of soluble nuclear fraction are reflected in the genome state. Although the genome is often depicted as a static structure upon which protein factors bind to control the turn of expression, the genome is actually highly mobile and capable of exploring the complex domain architecture of the nucleus, which in turn controls genome maintenance and gene expression. The impact of EHF EMI (Extremely High Frequencies Electromagnetic Irradiation) of the mm-range (45-53 $\mathrm{GHz}$ ) on protein, RNA, and DNA content in the composition of the nuclear envelope and a soluble nuclear fraction of wheat seedlings on the 4- day after irradiation have been studied. These modifications in nuclei content of seeds during growth after exposure by EMF may be related to abnormal photosynthetic activity, as well as with plant transcriptome activity, which more visible by study modulation in the content of 5-mC DNA methylation. The part of investigated seedlings was growing in soil to get a harvest of second-generation. Obtained in our study data shows that the changes in the level of DNA methylation in the first generation of seeds during the plant ontogeny are conserved and partially pass to the seedlings of second-generation seeds. So we have shown that changes in the content of nuclei and DNA methylation levels can be passed to the next generation and provide a memory that enables the plant and even its offspring to adapt better to subsequent stress through epigenetic mechanisms. 